\title{
El conocimiento escolar y las Hipótesis de Progresión: algunos fundamentos y desarrollos*
}

\author{
SCHOOL KNOWLEDGE AND THE PROGRESSION HYPOTHESIS: SOME FUNDAMENTALS AND \\ DEVELOPMENTS
}

O CONHECIMENTO ESCOLAR E AS HIPÓTESES DE PROGRESSÃO: ALGUNS FUNDAMENTOS

E DESENVOLVIMENTOS

\begin{abstract}
Carmen Alicia Martínez Rivera** / camartinezr@udistrital.edu.co
Vilma Graciela Martínez Rivera**/ vigramari@yahoo.es
\end{abstract}

\section{Resumen}

El conocimiento escolar es abordado como un conocimiento particular, en este artículo se presenta una mirada desde el contexto en el que éste se construye; pasando por identificar otras clases de conocimientos presentes en el contexto escolar, así como algunos referentes y dimensiones desde los cuales se soporta. Igualmente, se analizan algunas categorías que permiten estructurar este conocimiento, y su relación con las Hipótesis de Progresión y diferentes factores para tener en cuenta en su construcción, como por ejemplo, la propuesta que plantea Martínez respecto de los Ejes DOC. Este artículo concluye con la presentación de varios ejemplos de Hipótesis de Progresión, desarrollados en diferentes investigaciones y áreas de conocimiento. Destacando a la vez que las Hipótesis de Progresión se constituyen en una propuesta de gran potencialidad para la intervención e investigación didáctica

\section{Summary}

School knowledge is approached as a particular knowledge; this article presents a view from the context in which it is built, through identifying other kinds of knowledge present in the school context, as well as some referents and dimensions, from which supports. Also discusses some categories that allow to structure this knowledge and its relation to Progression Hypothesis and different factors to consider in its construction, for example, the proposal raised by Martinez for axes DOC. This article concludes by presenting several examples of Progression Hypothesis developed in different research studies and areas of knowledge. It is highlighted that Hypothesis of Progression, constitute a proposal with great potential for didactic intervention and research.
Palabras clave

Conocimiento escolar, Hipótesis de Progresión

\section{Keywords}

School knowledge, Hypothesis of Progression

\footnotetext{
* Elaborado en el marco del proyecto de investigación "El conocimiento profesional de los profesores de ciencias sobre el conocimiento escolar: dos estudios de caso en el Distrito Capital". CIDC. Universidad Distrital Francisco José de Caldas.

** Doctora en Educación-Didáctica de las Ciencias, Profesora en el Doctorado Interinstitucional en Educación de la Universidad Distrital Francisco José de Caldas; camartinezr@udistrital.edu.co, carmenaliciamartinezrivera@gmail.com

***Artista Plástica, Licenciada en Lingüística y Literatura; D.E.A. en Escultura e Historia del Arte e investigadora independiente: vigramari@yahoo.es
}

Fecha de recepción: 13 de mayo de 2012 / Fecha de aprobación: 8 de junio de 2012 
Resumo

O conhecimento escolar é abordado como um conhecimento particular, neste artigo se apresenta uma mirada desde o contexto no que este se constrói; passando por identificar outras classes de conhecimentos presentes no contexto escolar, bem como alguns referentes e dimensões desde os quais se suporta. Igualmente, analisam-se algumas categorias que permitem estructurar este conhecimento, e sua relação com as Hipóteses de Progressão e diferentes factores para ter em conta em sua construção, como por exemplo, a proposta que propõe Martínez respecto dos Eixos DOC. Este artigo conclui com a apresentação de vários exemplos de Hipóteses de Progressão, desenvolvidos em diferentes investigações e áreas de conhecimento. Destacando ao mesmo tempo que as Hipóteses de Progressão se constituem numa proposta de grande potencialidad para a intervenção e investigação didáctica.

Palavras chave

Conhecimento escolar, Hipótese de Progressão

\section{Introducción}

¿Qué hace que la escuela sea un espacio particular para el proceso de enseñanza-aprendizaje? ¿Por qué no es suficiente el conocimiento disciplinar para ejercer este proceso en un espacio como la escuela? Si bien son varios los aspectos que hacen del espacio escolar un contexto diferenciado en el proceso de formación, tendremos en cuenta para este artículo un aspecto central: el del conocimiento escolar y su relación con las Hipótesis de Progresión.

Con ello buscamos acercarnos a interrogantes como los enunciados, con la precisión de que la mirada que aquí exponemos, centrada en una perspectiva alternativa de formación, más que pretender ser una receta para seguir, es más que relevante, ya que funciona no sólo puente para plantear otras visiones que contribuyan a entender lo que pasa en el contexto particular de la escuela, sino también como mecanismo que permite dar opciones para enriquecer el camino que se está construyendo desde ésta.

\section{La escuela como contexto particular}

Sabemos que alrededor del espacio físico escolar y de los sujetos que intervienen en el proceso de enseñanzaaprendizaje, estudiantes y maestros, se tejen caminos, ideas, y otros eventos invisibles propios de las sociedades a las que pertenecen dichos espacios escolares. Esta particularidad de la escuela y sus tejidos, ha llevado a que autores como Cañal y Porlán (1987) vean estos espacios como un "sistema singular y complejo", determinado por los contextos con los que se relaciona, dada su condición de "sistema abierto".

Por ejemplo, el entorno del camino que diariamente recorren los alumnos de su casa al colegio; las complejidades propias de las instituciones, como: los núcleos familiares de cada niño, las particularidades y diversidades, tanto en formación disciplinar como personal, de los distintos docentes con los que ellos interactúan; así como las condiciones sociales, políticas, económicas y culturales de cada espacio y momento histórico vivido por el país; se convierten en mucho más que una sumatoria de complejidades, algunas invisibles otras no, que tarde o temprano se visibilizan como otras formas de saber que obligatoriamente intervienen en el tejido del conocimiento escolar. 
Así, formar y aprender termina siendo un proceso que se complejiza no sólo por algunas de las razones antes expuestas, sino por la necesidad de reestructurar pensamientos y actitudes tradicionales que planteaban únicas miradas o caminos para construir conocimiento. En un tejido tan diverso y rico como la escuela se "hacen construcciones particulares" (Chaparro, Orozco y Martínez, 1996).

Es en este "espacio vivo", multidiverso, intercultural y centro de producción de constantes y variables temas de investigación, donde se espera formar sujetos críticos, ávidos de conocimientos y pensamientos que contribuyan directamente a generar acciones favorables en sus particulares sociedades, tal como lo manifiesta Morín (1990) al aludir al papel de la educación respecto al conocimiento, en el que se debe: "[...] tener en cuenta el contexto, lo multidimensional, lo global, la interacción compleja; la incertidumbre, incluida la validez del conocimiento; no reducir el ser humano a una o varias de sus cualidades" (p.117). Maneja el mismo sentido cuando se refiere a la complejidad en la escuela, pues no debe aparecer como "una receta para conocer lo inesperado", pero sí requiere de que nos hagamos más "prudentes, atentos, y no dormirnos en la mecánica aparente y la trivialidad aparente de los determinismos" (Morín, 1990, p.117).

Podemos entonces ratificar de entrada que la escuela es un gran espacio generador de transformación social, lo que nos motiva, a los maestros, a participar más activamente en esta dinámica de construcción de sociedad. Esto requiere de que los actores que dinamizamos este espacio vital, nos reconozcamos y reconozcamos en el otro esa potencia y fuerza que nos habita, esa experiencia de vida que genera dinámicas particulares para entender su yo, su entorno y, por supuesto, su sociedad; es esta mirada una opción para entender la complejidad y la dureza de las realidades de nuestras comunidades educativas en sociedades tan particulares como las latinoamericanas y en especial la colombiana (Martínez y Martínez 2004).

Así como nos encontramos con grupos de estudiantes cuyas miradas de la vida están acompañadas de un relativo optimismo, de la misma manera encontramos otros, cuyos imaginarios se han transformado a partir de los desplazamientos forzosos que han vivido junto con sus comunidades, lo que los ha llevado a sufrir una serie de desprendimientos individuales, familiares y sociales, afectando y modificando su manera de ver el mundo y su existencia.

\section{La escuela y sus conocimientos}

Cuando nos preguntamos por el conocimiento que queremos trabajar en la escuela, tenemos que señalar que desde ésta se generan por lo menos dos conocimientos particulares, el conocimiento escolar, y el conocimiento profesional, conocimientos propios de las riquezas que se tejen en este espacio. La presencia de dichos conocimientos plantea el reconocimiento de una epistemología escolar particular, donde tanto el conocimiento escolar, como el conocimiento profesional del profesor, son dos conocimientos epistemológicamente diferenciados (García, 1998).

Estos reconocimientos se han dado también en el caso de la Didáctica, que ha dejado de ser asumida como un conjunto de técnicas, para considerarla como una disciplina (Gil, Carrascosa y Martínez, 2000; Porlán, 1998; Moreira, 2005; Tobin, 1998) con problemas y comunidades particulares, ligada a la construcción de nuevos objetos de estudio. Así la didáctica pasó de ser un adjetivo, para ser sustantivo, lo que implica que los contenidos de enseñanza deban ser no sólo construidos, sino elaborados a partir de conceptos particulares que den cuenta de estos procesos (Astolfi, 2001).

\section{Conocimiento escolar}

Es necesario destacar que distintos investigadores, desde la historia de la educación, ya se referían a las disciplinas escolares señalando las particularidades creativas en la escuela (Goodson, 1991; Chervel, 1991), también se ha planteado la necesidad de una transposición didáctica en el contexto de las matemáticas (Chevallard, 1991) y, desde la Didáctica de las Ciencias, se resalta como un gran reto la construcción de una Teoría del Conocimiento Escolar (Porlán, 1998; García, 1998; Giordan y De Vecchi, 1995; Izquierdo, 2005), que algunos Ilaman "Ciencia Escolar" (Izquierdo, 2005; Aduriz-Bravo, 2001).

Una sistematización de las investigaciones sobre el Conocimiento Escolar, socializadas en las versiones VI y VII del Congreso Internacional de Enseñanza de las Ciencias (2005 y 2009), y publicadas en la Revista 
Enseñanza de las Ciencias (Martínez, Molina y Reyes, 2010), nos permite señalar que los autores se refieren a este conocimiento de maneras variadas: Ciencia Escolar (Carrera, I. y Vierna, L., 2005; Bahamonde, N., y Pijol, R., 2009); Conocimiento Científico Escolar (Nardi, M., y Almeida, M., 2005); Conocimiento Escolar, (Martínez, C., y Rivero, A., 2005; Peme-Aranega, C., De Longhi, A., y Moreno, A. 2005); Contenidos Escolares (Almeida, N., y Riveiro, E., 2005); Conocimiento en la Escuela (Mojica, L., Molina, A., López, D., y Torres, B., 2005); Ciencia en Primaria (Cuéllar, L., Pérez, R., y Quintanilla, M., 2005); Saber Escolar (Texeira, A., y Krapas, S., 2005; Nardi, M., y Almeida, M.,2005); Currículo Escolar (Quadrado, R., y Ribeiro, P., 2005); en las que, a pesar de ser mayoritario el uso del término Ciencia Escolar, seguido de Conocimiento Escolar y Conocimiento Científico Escolar; todos parecen destacar su carácter específico, ya sea indicando que se presenta en un contexto particular, el escolar, o también resaltando un carácter científico.

Si bien son diversas las miradas frente a lo que se ha Ilamado conocimiento escolar, tratando de definir lo que entendemos por éste, consideramos pertinente la mirada que al respecto plantea García (1998, como: "[...] un conocimiento organizado y jerarquizado, procesual y relativo, como un sistema de ideas que se reorganiza continuamente en la interacción con otros sistemas de ideas, referidos a otras formas de conocimiento" (p. 151). En esta interacción intervienen no sólo los saberes disciplinares del profesor, sino también el conocimiento que tienen los estudiantes y el conocimiento cotidiano presente en ellos (Merchán J. y García F. 1996). La manera como se concreta este conocimiento escolar, según la propuesta de García es a través de Hipótesis de Progresión.

\section{Referentes para la construcción del conocimiento escolar}

Como bien decíamos, han sido distintas las maneras de entender y de asumir el conocimiento, esta diversidad se puede ver en algunas de las "tendencias" que se recogen en Martínez (2000, 2005a) a partir del proyecto IRES (Investigación y Renovación Escolar) del Grupo Investigación en la Escuela (1991) y de otros autores por ella citados:

- Perspectiva evolutiva y constructivista del conocimiento: desde esta perspectiva se considera que las personas conocemos a través de un proceso de interacción entre sus teorías, intereses, creencias, etc., y factores externos. (Toulmin, 1972; Novak, 1987; Porlán, García y Cañal, 1988, entre otros).

- Perspectiva sistémica y compleja del mundo: desde este enfoque se asume a las ideas y a la realidad como sistemas en evolución, en los que su complejidad está determinada por la cantidad y calidad de los elementos que la componen, así como de sus interacciones (Porlán y Rivero, 1998; Morín, 1977, 1982, 1986 y 1990; García, 1994, 1995a, 1995b; Cañal y Porlán, 1988). Desde esta perspectiva, cabe resaltar las necesarias relaciones del Conocimiento Escolar con la Cultura (Martínez y Molina, 2011).

- Perspectiva crítica: en ella se considera que el proceso de conocimiento no es un proceso neutral, sino que está influido por las propias maneras de ver el mundo y por sus intereses (Porlán y Rivero, 1998; Habermás, 1968, 1981; Carr y Kemmis, 1988; Apple, 1979).

\section{Dimensiones del conocimiento escolar}

En relación con las dimensiones del conocimiento escolar, nos permitimos destacar dos principios planteados por García (1994, p. 66) con los que pretende una visión integrada y compleja del conocimiento escolar, desde el reconocimiento de su carácter estructural y dinámico.

- Estructural: permite considerar al conocimiento escolar como "un conocimiento organizado y jerarquizado, como un sistema de ideas"; éste se corresponde a las tramas de contenidos de los profesores y a la explicación de las concepciones de los alumnos.

- Dinámico: permite precisar, que éste es un conocimiento con un "carácter procesual y relativo"; se corresponde a la elaboración de Hipótesis de Progresión y a sus niveles de formulación, en particular desde el interés didáctico, al cambio de los sistemas de ideas de los alumnos.

\section{Caracterización del conocimiento escolar}

Como lo hemos señalado, en Martínez (2005a), el conocimiento escolar se caracteriza por favorecer un proceso de transición que va desde una perspectiva simple hacia una compleja. Hemos planteado las si- 
guientes categorías como las más relevantes para dar cuenta del conocimiento escolar a partir del diseño de Martínez (2000)1:

- Tipos de contenidos: permiten dar cuenta si se considera una determinada variedad de contenidos, entre otros: conceptuales, actitudinales y procedimentales.

- Grados de generalidad: permiten comprender si se han previsto las tramas de contenidos o identificado contenidos estructurales para su organización.

- Fuentes y criterios de selección: abordan los aspectos que se tienen en cuenta para definir qué enseñar y qué actividades académicas o institucionales son consideradas para la formulación de contenidos.

- Referentes: en esta categoría se tienen en cuenta los tipos de conocimientos que son considerados en la construcción del conocimiento escolar.

- Relaciones horizontales: hacen referencia a la identificación, tanto del tipo de organización, como del número y relación de los diferentes elementos presentes en los contenidos.

- Niveles de formulación: permiten comprender los cambios graduales en la construcción del conocimiento escolar.

- Criterios de validez: determinan desde dónde se define qué es o no adecuado frente al conocimiento escolar.

Algunos de los resultados de nuestros trabajos permiten señalar la relevancia de las categorías: fuentes y criterios de selección, referentes y criterios de validez (Martínez y Rivero, 2001); así como diferentes relaciones entre las consideraciones sobre el conocimiento científico y el conocimiento escolar, que pone en cuestionamiento una relación causal entre estos dos conocimientos (Martínez y Rivero, 2009).

\section{Las Hipótesis de Progresión como propuesta de organización del conocimiento escolar}

En el proceso de organización del conocimiento escolar, las Hipótesis de Progresión y sus sistemas de ideas, aparecen como una alternativa para intentar encaminar

\footnotetext{
1 De manera más detallada, presentamos algunos resultados en relación
} con estas categorías, en Martínez y Rivero (2001, 2005 y 2009). de manera argumentada, explícita y reflexionada, las propuestas de enseñanza. En este sentido una Hipótesis de Progresión, según García (1998), hace referencia: "[...] tanto a un contenido concreto (la construcción gradual y progresiva de una determinada idea) como a un conjunto de contenidos conectados entre sí en una trama (representación curricular del cambio en la organización de un sistema de ideas)".

Esta manera progresiva de abordar las hipótesis de lo simple a lo complejo, parte de asumirlas como una estrategia didáctica desde IRES, dado el predominio de un pensamiento simple, y la necesidad de hacer un proceso de transición, para llegar a un pensamiento complejo (García, 1995a, 1995b; García, Martín y Rivero, 1996; García y Merchán, 1997; Porlán, 1993; Porlán y Rivero, 1998; García P., 1999). Al construir las hipótesis de manera gradual y evolutiva, se van determinando niveles de formulación cuya diferenciación sirve de marco de referencia y de orientación en los procesos dinámicos de enseñanza y aprendizaje (Giordan y De Vecchi, 1995; García, 1994).

Lo anterior, implica una perspectiva evolutiva del conocimiento escolar en la que no hay una "destrucción", sino una "transformación" orientada por la Hipótesis de Progresión; a modo de "teorías provisionales" con campos de validez determinados que requieren de ser precisados por el enseñante (Giordan y De Vecchi, 1995).

\section{Aspectos a tener en cuenta en la construcción de Hipótesis de Progresión}

Las Hipótesis de Progresión se constituyen no sólo como un referente para la investigación, para la comprensión de la construcción del conocimiento en la escuela, sino además para orientar de manera fundamentada los procesos de enseñanza, así como las propuestas de formación de profesores. Sin embargo, es necesario anotar que aunque las Hipótesis de Progresión se organizan, como su nombre lo señala, en una perspectiva progresiva, éstas además de orientar, posibilitan entender los procesos de transformación sin indicar una perspectiva lineal del proceso, sino que, por el contrario, se asumen de una manera flexible en la que son posibles ciclos o fluctuaciones propias de los procesos de formación (Porlán y Rivero, 1998).

Así organizadas, las Hipótesis de Progresión permiten, de manera fundamentada, comprender la complejidad 
del conocimiento escolar, en tanto que desde diferentes niveles, contenidos o categorías, es posible orientar o comprender momentos particulares en el proceso educativo. Así por ejemplo, resultados de nuestras investigaciones muestran que dependiendo, tanto del aspecto analizado, como del momento dado, se puede caracterizar una tendencia como cercana a un nivel de complejidad o a otro, quedando claro que no todos los aspectos analizados se identifican en el mismo nivel de complejidad (Martínez y Rivero, 2009).

Dentro del proceso de construcción de las Hipótesis de Progresión, se requiere de la investigación como soporte del proceso de formulación de las hipótesis, especialmente "en relación con la construcción de conocimientos a través de niveles de formulación progresivamente más complejos y elaborados" (Merchán y García, 1996). Además, es necesario el problema, como pauta de investigación, pues permitirá al docente plantearse preguntas claves como: "qué enseñar", "cómo enseñar" y cómo y qué "evaluar". En el caso de los alumnos, el planteamiento de preguntas, les posibilitará niveles de respuestas que se complejizarán en la medida en que se vayan reformulando sus problemas, generando así niveles de profundidad y de complejidad en la construcción de conocimientos. En este sentido, el planteamiento de los problemas se convierte en aspecto fundamental para la elaboración de los distintos niveles de formulación (Astolfi, 2001).

A continuación, enunciamos algunos aspectos para tener en cuenta en el proceso de construcción de $\mathrm{Hi}$ pótesis de Progresión:

- Organización que vaya de perspectivas simples a perspectivas complejas.

- Constante revisión y reformulación.

- Construcción progresiva y evolutiva.

- Evidencia del proceso de transformación de las ideas.

- Disposición de posibles itinerarios de manera abierta y flexible.

- Elaboración, tanto de niveles de formulación, como de profundidad. En este sentido García (1999), identifica los siguientes niveles: el paso de un aprendizaje "aditivo simple" a un aprendizaje "aditivo complejo", y a su vez, a un aprendizaje por "descubrimiento espontáneo", pasando a un aprendizaje "constructivista simple", para llegar a un aprendizaje "constructivista complejo".

- Paso de un conocimiento cotidiano y simple a un conocimiento más riguroso y complejo, un proceso en el que las ideas de los alumnos no se vean como errores, sino que, por el contrario, se reconozcan como parte del proceso del alumno, para que a su vez permitan la interacción de ellos con otros sujetos y con su medio (García, 1999).

Al respecto, es pertinente señalar como los profesores construyen un conocimiento que les posibilita dar cuenta de esos diferentes niveles de complejidad, como parece es el caso de la profesora Nancy, mencionada por Shulman (2001), de quien comenta cómo alude a los diferentes niveles de las habilidades de lectura, así: ella parte con una primera fase que consiste en la traducción, con la que se busca entender el significado literal, en un nivel denotativo; pasando por una compresión del significado connovativo, en un nivel de interpretación; y llegar a un nivel más complejo, con el que se pretende comprender el sentido de la literatura.

[...] mi visión de la lectura consiste básicamente en lograr que los alumnos pasen desde el contenido literal que aparece en la página hasta hacer que éste tenga algún significado en sus vidas. Al enseñar literatura siempre estoy entrando y saliendo de estos niveles (Gudmundsdottir, citado por Shulman, 2001, p. 165).

Con este ejemplo podemos ver cómo uno de los aspectos centrales en la construcción de las Hipótesis de Progresión, es la comprensión de la manera como los profesores han venido construyendo su conocimiento profesional en los contextos particulares.

\section{Incidencia de los Ejes DOC en la construcción y desarrollo de las Hipótesis de Progresión}

Dada la mirada progresiva y transformadora de las Hipótesis de Progresión en el proceso de enseñanza y aprendizaje, consideramos pertinente hacer referencia a la categoría de Ejes DOC (Dinamizadores, Obstáculo y Cuestionamiento), propuesta por Martínez (2000). Desde la cual no sólo se cuestiona la concepción de obstáculo y error, aplicada a las ideas previas de los alumnos, sino que, por el contrario, se plantea la necesidad de transformar sin destruir o desconocer estos aportes iniciales de los estudiantes. Conviene hacer 
una mirada que no abarque únicamente el punto de vista de los ejes obstáculos, sino que incluya además, los ejes movilizadores y de cuestionamiento. Esto es, indagar en las ideas de los alumnos, por aquello que impide o que, en un momento dado, promueve y dinamiza, e incluso por aquello que está en dicotomía o en divergencia.

En este sentido, lo que hemos considerado durante mucho tiempo como un obstáculo, pasa, desde la perspectiva de la complejidad, a ser un eje dinamizador. Así, las ideas de los alumnos no se identifican como errores sino como bases para la construcción de nuevos conocimientos:

Si se toma como referencia una concepción constructivista del aprendizaje hay que admitir que éste se produce por interacción entre el conocimiento de que dispone el alumno y las nuevas informaciones que le llegan. Ello obliga a considerar las concepciones de los alumnos no como "conocimientos erróneos" (por el hecho de que no se ajusten a lo que se considera correcto, desde el punto de vista científico), sino como bases o puntos de engarce sobre los que se irán construyendo los nuevos conocimientos (García y García, P., 1989, p.40).

Es a la influencia positiva que favorece el cambio a lo que se denomina eje dinamizador o movilizador. Autores como Toulmin (1972) ya reconocían esa complejidad en la evolución conceptual al señalar la existencia de lo que Ilama "variantes exitosas, variantes abortivas y variantes bisoñas, en debate". En este sentido Astolfi (1999) invita a reconocer una cierta complementariedad en dos autores clásicos: Bachelard, quien con la mirada sobre los obstáculos, ofrece un modelo "Regresivo y "pesimista", y Piaget, cuyo modelo es "prospectivo y "optimista", y propone el "tratamiento didáctico estratégico", que mira más hacia delante que hacia atrás, pasando de las condiciones requeridas hacia las condiciones de posibilidad.
Así, los ejes Dinamizador, Obstáculo, y Cuestionamiento, ejes DOC, propuestos por Martínez (2000), y adoptados por Ballenilla (2003) y Solís (2006), se plantean como categorías de análisis para comprender el conocimiento de los profesores en general, e inevitables para el caso particular del conocimiento escolar. Estos ejes son entendidos así: el Eje Obstáculo, que comprende el conocimiento del profesor en medio de una relación que aunque le posibilita su actuar no favorece el desarrollo profesional; el Eje cuestionamiento, que alude a conocimientos alternativos que sin embargo resultan insuficientes para orientar la acción, evidenciando contradicciones; y el Eje Dinamizador, es decir, conocimientos que se aproximan a visiones alternativas, y que al parecer impulsan y catalizan un proceso de cambio.

Los trabajos realizados nos señalan que, respecto al conocimiento escolar, hay una gran incidencia de las consideraciones que hemos realizado acerca de estas tres categorías, no sólo en el sentido de que se configuran como un espacio en el que pueden encontrarse las principales dificultades del proceso, y desde allí encontrar posibles "fisuras" para emprender el cambio, sino como contexto ideal para favorecer el desarrollo profesional (Martínez, 2000). Es por ello que consideramos que es posible avanzar en una comprensión que intente abordar la complejidad de las anteriores categorías, para el análisis de estos conocimientos particulares en la escuela, si no sólo las adoptamos, sino que las consideramos dentro de su integridad.

\section{Ejemplos de Hipótesis de Progresión}

En este apartado señalaremos algunos ejemplos de Hipótesis de Progresión. Es posible ubicar diferentes investigaciones y propuestas didácticas que las han desarrollado, tanto en relación con la formación de profesores, como con el conocimiento escolar (Tabla 1), y en diferentes áreas (enseñanza de la ecología, enseñanza de la química, enseñanza de la biología y de la educación ambiental). 


\begin{tabular}{|c|c|c|}
\hline Hipótesis de Progresión & Autores & Descripción \\
\hline $\begin{array}{l}\text { Propuesta curricular de Hipótesis de Progresión } \\
\text { para conceptos estructurantes de ecología }\end{array}$ & $\begin{array}{l}\text { Bermúdez y De Longhi } \\
\qquad(2006)\end{array}$ & $\begin{array}{c}\text { Propuesta curricular para abordar los diferentes } \\
\text { niveles de complejidad de conceptos estructu- } \\
\text { rantes en ecología. Diseño de unidad didáctica } \\
\text { para el trabajo con alumnos }\end{array}$ \\
\hline $\begin{array}{l}\text { Hipótesis de Progresión de las posibles evolucio- } \\
\text { nes de las actitudes, } \\
\text { percepciones y concepciones sobre medio am- } \\
\text { biente y riesgos ambientales }\end{array}$ & Morón y Wamba (2010) & $\begin{array}{l}\text { Estudio con Estudiantes de pedagogía y estudian- } \\
\text { tes de ciencias ambientales. }\end{array}$ \\
\hline $\begin{array}{l}\text { Una Hipótesis de Progresión sobre los modelos } \\
\text { de desarrollo en Educación Ambiental }\end{array}$ & García (1999) & $\begin{array}{c}\text { Estudio de las concepciones en estudiantes del } \\
\text { magisterio. (Investigación realizada en España, } \\
\text { en donde los estudiantes de magisterio realizan } \\
\text { estudios universitarios para la enseñanza en } \\
\text { primaria) }\end{array}$ \\
\hline $\begin{array}{l}\text { Hipótesis de Progresión a cerca } \\
\text { del conocimiento biológico }\end{array}$ & & \\
\hline $\begin{array}{l}\text { Hipótesis de Progresión acerca del conocimiento } \\
\text { didáctico del contenido biológico }\end{array}$ & Valbuena (2007) & $\begin{array}{l}\text { Estudio del conocimiento didáctico del conteni- } \\
\text { do biológico de los futuros docentes de biología. }\end{array}$ \\
\hline $\begin{array}{c}\text { Una propuesta de conocimiento } \\
\text { profesional deseable sobre la enseñanza } \\
\text { de los contenidos escolares }\end{array}$ & \multirow{3}{*}{ Martín (1994) } & \multirow{3}{*}{$\begin{array}{c}\text { Estudio de las concepciones disciplinares y } \\
\text { didácticas de los estudiantes de magisterio sobre } \\
\text { el cambio químico (Investigación realizada en } \\
\text { España) }\end{array}$} \\
\hline Niveles de formulación del cambio químico & & \\
\hline $\begin{array}{l}\text { Niveles de formulación en las concepciones de } \\
\text { los alumnos sobre los cambios químicos }\end{array}$ & & \\
\hline $\begin{array}{c}\text { Hipótesis de Progresión en torno a: concepcio- } \\
\text { nes curriculares del profesorado sobre objetivos } \\
\text { educativos, formulación de contenidos escola- } \\
\text { res, concepciones e intereses del alumnado, y } \\
\text { evaluación }\end{array}$ & Solís (2005) & $\begin{array}{c}\text { Concepciones curriculares de profesores de quí- } \\
\text { mica y física en formación inicial. (Investigación } \\
\text { realizada en España con profesionales titulados } \\
\text { en Licenciatura en química, o en física) }\end{array}$ \\
\hline $\begin{array}{l}\text { Hipótesis de Progresión en la metodología para } \\
\text { enseñar ciencias }\end{array}$ & Porlán y Martín (2005) & $\begin{array}{c}\text { Concepciones sobre la secuencia de actividades } \\
\text { que se propone para enseñar contenidos concre- } \\
\text { tos en futuros maestros }\end{array}$ \\
\hline $\begin{array}{l}\text { Hipótesis de Progresión profesional sobre la } \\
\text { enseñanza-aprendizaje de las ciencias }\end{array}$ & Martin y Rivero (2001) & $\begin{array}{l}\text { Propuesta de formación de profesores de cien- } \\
\text { cias para la educación secundaria, por ámbitos } \\
\text { de investigación profesional }\end{array}$ \\
\hline $\begin{array}{l}\text { Hipótesis de Progresión sobre las ideas de los } \\
\text { alumnos }\end{array}$ & García (1999) & $\begin{array}{c}\text { Propuesta de Hipótesis de Progresión } \\
\text { y dificultades para el desarrollo profesional de } \\
\text { los profesores }\end{array}$ \\
\hline
\end{tabular}

Tabla 1. Ejemplos de propuestas de Hipótesis de Progresión 
Los ejemplos de Hipótesis de Progresión que se presentan a continuación, han sido elaborados en áreas del conocimiento como ecología y artes. Dentro de estas áreas, García (1997) propone una Hipótesis de Progresión para la construcción del concepto de ecosistema, organizada en tres niveles de formulación y en cuatro contenidos básicos, que recogemos en la Tabla 2.

\begin{tabular}{|c|c|c|c|}
\hline \multirow[b]{2}{*}{ Contenidos } & \multicolumn{3}{|c|}{ Niveles de formulación } \\
\hline & $\begin{array}{l}\text { 1. Ideas previas esperadas,, } \\
\text { concepción aditiva }\end{array}$ & $\begin{array}{l}\text { 2. Nivel de transición, perspecti- } \\
\text { va más sistémica }\end{array}$ & $\begin{array}{l}\text { 3. Nivel de mayor compleji- } \\
\text { dad, incluye nociones sisté- } \\
\text { micas }\end{array}$ \\
\hline $\begin{array}{l}\text { Contenido } 1 \\
\text { Categorización y } \\
\text { descripción de ele- } \\
\text { mentos y relacio- } \\
\text { nes presentes en el } \\
\text { ecosistema }\end{array}$ & $\begin{array}{l}\text { Énfasis en el meso-cosmos: } \\
\text { se reconocen animales } \\
\text { prototípicos y las acciones } \\
\text { de los seres vivos, más no } \\
\text { su papel organizador en la } \\
\text { estructura del ecosistema }\end{array}$ & $\begin{array}{c}\text { Se reconoce la diversidad de } \\
\text { seres vivos y de producciones } \\
\text { de los mismos, indicando su } \\
\text { presencia en los ecosistemas. } \\
\text { Se admite que las relaciones } \\
\text { ecológicas se dan a nivel de } \\
\text { poblaciones }\end{array}$ & $\begin{array}{l}\text { Se reconocen componentes } \\
\text { poco evidentes del ecosiste- } \\
\text { ma: seres microscópicos; se } \\
\text { asume mayor complejidad y } \\
\text { diversidad en las relaciones en } \\
\text { el ecosistema }\end{array}$ \\
\hline $\begin{array}{l}\text { Contenido } 2 \\
\text { Organización } \\
\text { del ecosistema y } \\
\text { grado de com- } \\
\text { plejidad de las } \\
\text { configuraciones } \\
\text { generadas por } \\
\text { las relaciones } \\
\text { ecológicas }\end{array}$ & $\begin{array}{c}\text { Se reconocen relaciones } \\
\text { sencillas, binarias; se trata } \\
\text { de una organización aditiva } \\
\text { del ecosistema, determina- } \\
\text { da por los elementos que } \\
\text { lo constituyen, no por sus } \\
\text { relaciones }\end{array}$ & $\begin{array}{c}\text { Primera aproximación al con- } \\
\text { cepto de nicho ecológico a } \\
\text { través de una jerarquía trófica. } \\
\text { Primera aproximación a proce- } \\
\text { sos que subyacen a relaciones } \\
\text { como la fotosíntesis y la respi- } \\
\text { ración }\end{array}$ & $\begin{array}{l}\text { Se considera una red de } \\
\text { interacciones en la que todo } \\
\text { está conectado con todo. Se } \\
\text { reconoce la interdependencia } \\
\text { entre los componentes de un } \\
\text { ecosistema }\end{array}$ \\
\hline $\begin{array}{l}\text { Contenido } 3 \\
\text { Estabilidad y } \\
\text { cambio en el } \\
\text { ecosistema }\end{array}$ & $\begin{array}{l}\text { La estabilidad del sistema } \\
\text { está dada por factores di- } \\
\text { ferentes a su propia orga- } \\
\text { nización; no se admite el } \\
\text { cambio, cuando se admite, } \\
\text { éste se refiere a cambios en } \\
\text { el número de elementos, } \\
\text { no a la organización del } \\
\text { ecosistema }\end{array}$ & $\begin{array}{l}\text { Aproximación a una visión más } \\
\text { flexible del orden. La organiza- } \\
\text { ción en cadena sigue la "ley del } \\
\text { todo o nada"; la organización } \\
\text { depende de los elementos más } \\
\text { que de sus relaciones }\end{array}$ & $\begin{array}{l}\text { El ecosistema se mantiene } \\
\text { estable debido a su carácter de } \\
\text { sistema abierto. Concepción } \\
\text { flexible del cambio de los eco- } \\
\text { sistemas, equilibrios dinámi- } \\
\text { cos, reorganización continúa } \\
\text { del sistema }\end{array}$ \\
\hline $\begin{array}{l}\text { Contenido } 4 \\
\text { Lugar de la especie } \\
\text { humana en el } \\
\text { ecosistema }\end{array}$ & $\begin{array}{l}\text { Se considera a la naturaleza } \\
\text { como una entidad al servicio } \\
\text { de los humanos; si hay una } \\
\text { consideración respecto de los } \\
\text { límites en el uso, está relacio- } \\
\text { nada con visiones fatalistas y } \\
\text { catastróficas o de sobrevalora- } \\
\text { ción del papel de la tecnología } \\
\text { en la resolución de problemas } \\
\text { ambiéntales }\end{array}$ & $\begin{array}{l}\text { Consideración del papel que la } \\
\text { especie humana ocupa un determi- } \\
\text { nado nicho ecológico. Noción de } \\
\text { recursos limitados, reconocimien- } \\
\text { to de la problemática ambiental. } \\
\text { Aproximación a posiciones cercanas } \\
\text { al desarrollo sostenible o al ecolo- } \\
\text { gismo social }\end{array}$ & $\begin{array}{l}\text { Consideración de la superviven- } \\
\text { cia de la especie humana asocia- } \\
\text { da a problemas ambientales. Se } \\
\text { pretende asumir principios éticos } \\
\text { como la solidaridad planetaria. } \\
\text { El tratamiento de problemas } \\
\text { ambientales es inseparable de los } \\
\text { problemas sociales }\end{array}$ \\
\hline
\end{tabular}

Tabla 2. Hipótesis de Progresión para la enseñanza de la ecología

En el campo del arte, Ávila (1999) propone una Hipótesis de Progresión con tres niveles de formulación en torno a la secuenciación de contenidos relativos a los Fenómenos Histórico-Artísticos (H/A): 1) Hacia una capacidad progresiva para leer los fenómenos $\mathrm{H} / \mathrm{A} ; 2$ ) Hacia una capacidad progresiva para explicar los fenómenos $\mathrm{H} / \mathrm{A}$, y 3) Hacia una visión compleja de los fenómenos H/A. En este sentido, Martínez y Fernández (2000), ejemplifican dichos niveles de formulación en el caso particular de la obra "El Beso", del artista Augusto Rodin (Tabla 3). 


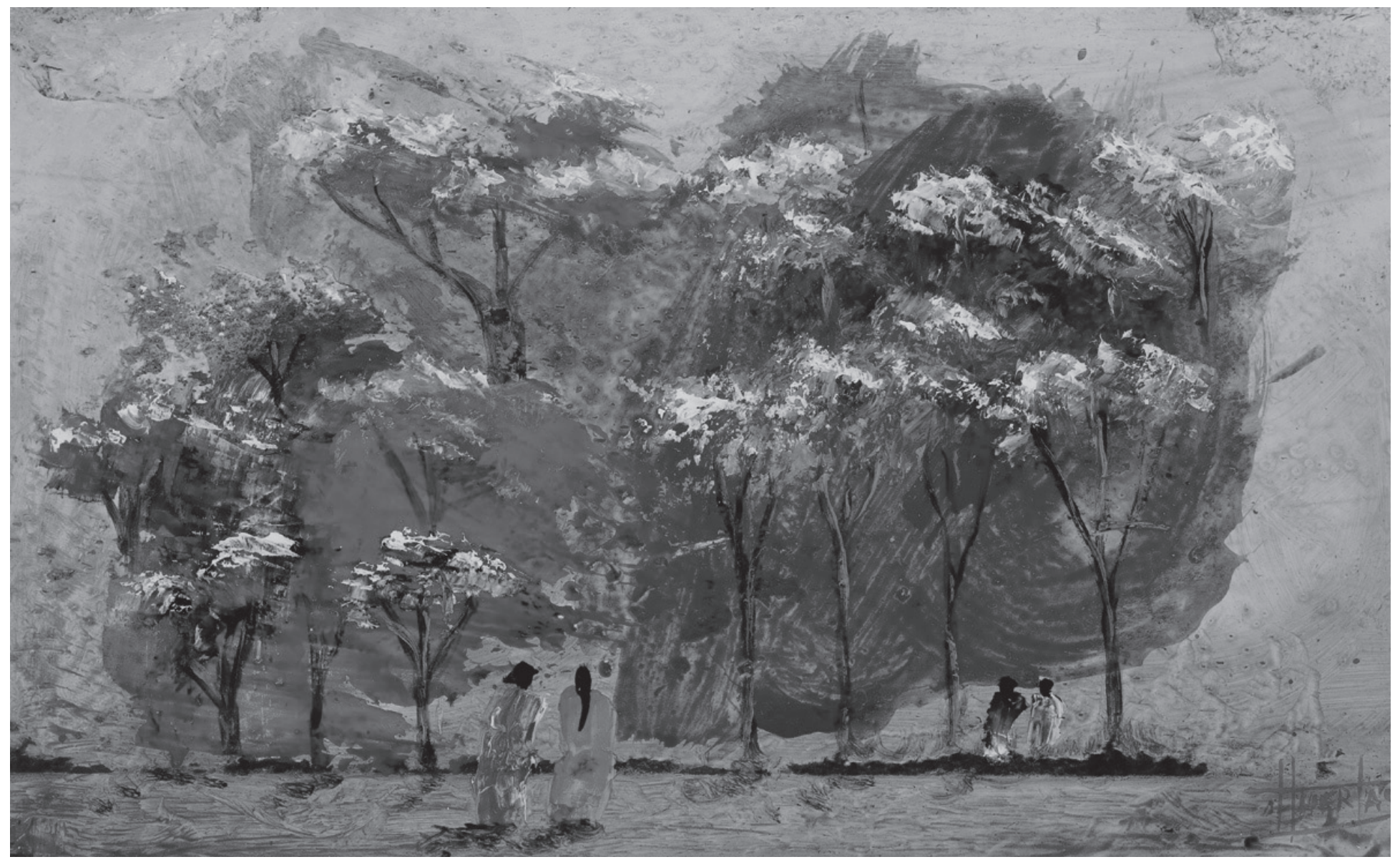

Segundo Arístides Huertas Torres ॥ Título: Bosque de ensueño ॥Técnica: Óleo sobre tela ॥ Dimensiones: $110 \mathrm{~cm}$ x $160 \mathrm{~cm}$ 


\begin{tabular}{|c|c|c|c|}
\hline Nivel de formulación & Contenido 1 & Contenido 2 & Contenido 3 \\
\hline $\begin{array}{l}\text { 1.Hacia una progresiva } \\
\text { capacidad para leer los } \\
\text { fenómenos H/A }\end{array}$ & $\begin{array}{c}\text { Primera aproximación } \\
\text { temática: dos personajes } \\
\text { besándose }\end{array}$ & $\begin{array}{l}\text { Utilización del mármol: } \\
\text { peculiaridades técnicas } \\
\text { Elementos secundarios: } \\
\text { libro }\end{array}$ & $\begin{array}{l}\text { Realizado por Augusto } \\
\text { Rodin, escultor francés, a } \\
\text { finales del siglo XIX; } \\
\text { presenta diferencias signi- } \\
\text { ficativas en el tratamiento } \\
\text { de los dos cuerpos }\end{array}$ \\
\hline $\begin{array}{l}\text { 2. Hacia una progresiva } \\
\text { capacidad para explicar } \\
\text { los fenómenos H/A }\end{array}$ & $\begin{array}{c}\text { La iniciativa tomada por la } \\
\text { mujer, refleja la particular } \\
\text { relación de Rodin con el } \\
\text { género femenino. Influencia } \\
\text { del contexto social, político } \\
\text { y económico }\end{array}$ & $\begin{array}{c}\text { La obra es encargada por } \\
\text { el Estado para el Museo de } \\
\text { Artes Decorativas. } \\
\text { La crítica cambia el título } \\
\text { por el del Beso }\end{array}$ & $\begin{array}{c}\text { Intencionalidad al usar } \\
\text { el lenguaje simbolista o } \\
\text { impresionista. Tema pro- } \\
\text { vocado por la sociedad y } \\
\text { la propia experiencia del } \\
\text { autor }\end{array}$ \\
\hline $\begin{array}{l}\text { 3. Hacia una visión com- } \\
\text { pleja de los fenómenos } \\
\text { H/A }\end{array}$ & $\begin{array}{c}\text { Representación de tres ejes } \\
\text { temáticos: pecado, beso, } \\
\text { amantes, ¿la obra es un } \\
\text { reflejo de la personalidad } \\
\text { del autor y de su visión de } \\
\text { la mujer?, ¿es una nueva } \\
\text { representación del pecado } \\
\text { original?, ¿es Francesca una } \\
\text { analogía de Eva? }\end{array}$ & $\begin{array}{c}\text { Comparación con sus } \\
\text { contemporáneos. Papel } \\
\text { de Rodin y su obra en el } \\
\text { contexto H/A: ¿es iniciador } \\
\text { de la escultura moderna? } \\
\text { Choque con el academicis- } \\
\text { mo de la Escuela de Artes }\end{array}$ & $\begin{array}{c}\text { Evolución de la personali- } \\
\text { dad y de la obra de Rodin } \\
\text { en relación con su contex- } \\
\text { to histórico y artístico. } \\
\text { Dificultad para inscribirlo } \\
\text { en un movimiento artístico } \\
\text { concreto. Valoración de la } \\
\text { obra del artista }\end{array}$ \\
\hline
\end{tabular}

Tabla 3. Hipótesis de Progresión en la obra "El Beso" del escultor francés Augusto Rodin

Los anteriores ejemplos ponen en evidencia un proceso de organización del conocimiento escolar que va de perspectivas simples, hasta llegar a perspectivas complejas; según los casos específicos se pasa, por ejemplo, de un énfasis en el meso-cosmos, a la consideración de otras perspectivas como el microcosmos y el macrocosmos; de relaciones aditivas al reconocimiento de interacciones; de una perspectiva antropocéntrica a una biorelativa; del énfasis en descripciones de formas artísticas, hacia la construcción de posibles explicaciones de los fenómenos artísticos; de la relevancia de elementos formales técnicos e iconográficos, hacia la consideración de la diversidad en el tiempo y el espacio; de la relación centrada en los elementos formales, hacia la contextualización de los fenómenos históricoartísticos como sistemas dinámicos. De esta manera, las Hipótesis de Progresión se afirman como un aporte significativo en la comprensión de las particularidades de los procesos escolares en su complejidad.

\section{Conclusiones}

El espacio escolar es un espacio particular en el que se desarrollan diferentes tipos de conocimientos, disciplinar, cotidiano y escolar, éste último lo abordamos desde el sentido de la construcción de Hipótesis de Progresión, que aparecen como aporte de gran potencial para las propuestas de enseñanza en las diferentes áreas del saber, ya que no sólo favorecen las visiones relativas del conocimiento, sino que además contribuyen en la organización progresiva del conocimiento escolar, promueven el enriquecimiento de las ideas iniciales de las personas en procesos de formación, y orientan tanto propuestas de investigación, como procesos de enseñanza y aprendizaje, desde una perspectiva fundamentada que busca abordar una perspectiva compleja de estos procesos.

Dadas las particularidades de los contextos en los que se inscriben las escuelas, las Hipótesis de Progresión 
son marcos de referencia, no sólo para interpretar y comprender los procesos escolares, sino para intervenir en ellos; constituyéndose como una orientación en el proceso formativo, y un mecanismo que además permite la organización de los referentes para comprender la complejidad del conocimiento del profesor y del conocimiento escolar.

Es por ello que consideramos pertinente continuar con la elaboración de este tipo de propuestas, así como con su experimentación, de modo que sea posible recurrir a experiencias didácticas que posibiliten el enriquecimiento mutuo del conocimiento que construimos en las instituciones educativas, en nuestros contextos particulares.

Se hace necesario, sin embargo, que estas propuestas se asuman en el contexto de la investigación, para evitar, de esta manera, que sean consideradas dentro de una perspectiva tecnológica que las Ileve a una aplicación acrítica. En este sentido, resulta inevitable destacar algunas investigaciones recientes que realizan aportes significativos en la construcción de Hipótesis de Progresión, tales como las adelantadas por Martínez C., Valbuena, Molina, y Hedereich (2009 y 2011) Hipótesis de Progresión del conocimiento profesional del profesor de ciencias sobre el conocimiento escolar. Junto a este trabajo habrá que mencionar también dos Tesis Doctorales, en curso, que contribuyen en la construcción de nuevas Hipótesis de Progresión: El conocimiento didáctico del contenido de profesores en formación sobre el concepto de Campo Eléctrico (Reyes, 2009; y Reyes y Martínez, 2011), y Las concepciones sobre la vida silvestre en estudiantes universitarios (Rubiano, 2010; Rubiano y Martínez, 2011).

\section{Referencias}

Adúriz-Bravo, A. (2001). Integración de la epistemología en la formación del profesorado de ciencias. Bellaterra: UAB.

Almeida, N., y Ribeiro, E. (2005). Projetos temáticos como alternativa para um ensino contextualizado das ciências: análise de um caso. Enseñanza de las ciências. (Número Extra). Obtenido el 2 de Octubre de 2009, desde, http://www.blues.uab.es/ sice23/ congres2005/material/comuni_orales/2_Proyectos_Curri/2_1/ almeida_812.pdf

Apple, M. (1979). Ideología y currículum. Routledge y Kegan Paul (Trad.) (1986). Madrid: Akal.

Astolfi, J. (1999). El "error", un medio para enseñar. Sevilla: Díada Editora.

Astolfi, J. (2001). Didáctica. Conceptos clave en la didáctica de las disciplinas. Sevilla: Díada.
Avila, R. (1999). La construcción del conocimiento escolar. Una propuesta de secuenciación de contenidos para la enseñanza de la historia del arte. Investigación en la escuela, 39, 39-48.

Bahamonde, N., y Pujol, R. (2009) Un enfoque teórico para el abordaje de temas complejos en el aula: el caso de la alimentación humana. Enseñanza de las Ciencias (Número Extra). Obtenido el 2 de Octubre de 2009, desde, http://ensciencias. uab.es/congreso09/numeroextra/art-2315-2319.pdf, 2315-2319.

Ballenilla, F. (2003). El prácticum en la formación inicial del profesorado de ciencias de enseñanza secundaria. Estudio de caso. Tesis doctoral no publicada. Universidad de Sevilla.

Bermúdez, G., y De Longhi, A. (2006). Propuesta curricular de hipótesis de progresión para conceptos estructurantes de ecología. Campo Abierto, 25 (2), 13-38. Obtenido el 3 de julio de 2007, desde, www.doredin.mec.es/documentos/00920073000037.pdf

Cañal, P. (1988). Un marco curricular en el modelo sistémico investigativo. En Porlán, R., García, J. E., y Cañal, P. (Comps.), Constructivismo y enseñanza de las ciencias. Sevilla: Díada.

Cañal, P., y Porlán, R. (1988). Bases para un programa de investigación en torno a un modelo didáctico de tipo sistémico e Investigativo. Enseñanza de las Ciencias, 6 (1), 54-60.

Carr, W., y Kemmis, S. (1988). Teoría crítica de la enseñanza. La Investigación en la acción en la formación del profesorado. Barcelona: Martínez Roca.

Carrera, I., y Vierna, L. (2005). Propuesta para incluir en los programas de "ciencia escolar", el tema uso responsable de los medicamentos. Enseñanza de las ciencias (Número Extra). Obtenido el 2 de Octubre de 2009, desde, http://ensciencias.uab. es/webblues/www/congres2005/material/comuni_orales/2_Proyectos_Curri/2_1/carrera_304.pdf

Chaparro, C., Orozco, J. y Martínez, C. (1996). Pensar la escuela ¿Política educativas vs Proyecto Educativo Institucional? Nodos y Nudos, 2, 4-12.

Chervel, A. (1991). Historia de las disciplinas escolares. Reflexiones sobre un campo de reflexión. Revista de Educación, 295 (I), 69 y 111.

Chevallard. (1991). ¿Qué es la transposición didáctica? La transposición didáctica: del saber sabio al saber enseñado. Argentina: Aique, 45-47.

García, J. E. (1994). El conocimiento escolar como un proceso evolutivo: aplicación al conocimiento de nociones ecológicas. Investigación en la Escuela, 23, 65-76.

García, J. E. (1995a). Proyecto docente. Sevilla: Universidad de Sevilla.

García, J. E. (1995b). Epistemología de la complejidad y enseñanza de la Ecología. El concepto de ecosistema en la educación secundaria. Tesis doctoral no publicada. Universidad de Sevilla.

García, J. E. (1995c). La transición desde un pensamiento simple hacia un pensamiento complejo en la construcción del conocimiento escolar. Investigación en la Escuela, 27, 7-20.

García, J. E. (1997). La formulación de hipótesis de progresión para la construcción del conocimiento escolar: una propuesta de secuenciación en la enseñanza de la ecología. Alambique, Didáctica de las Ciencias Experimentales, 14, 37-48.

García, J. E. (1998). Hacia una teoría alternativa sobre los contenidos escolares. Sevilla: Díada. 
García, J. E. (1999). Las ideas de los alumnos. Cuadernos de Pedagogía, 276, 58-64.

García, J. E., y García, F. F. (1989). Aprender Investigando. Una propuesta metodológica basada en la investigación. Sevilla: Díada Editora. Tercera edición, 1995.

García, J. E., Martín, J., y Rivero, A. (1996). El currículum integrado: desde un pensamiento simple hacia uno complejo. Aula de Innovación Educativa, 51, 13-18.

García, J. E., y Merchán, J. (1997). El debate de la interdisciplinariedad en la ESO: el referente metadisciplinar en la determinación del conocimiento escolar. Investigación en la Escuela, 32, 5-26.

García, J. E., y Rivero, A. (1996). La transición desde un pensamiento simple hacia otro complejo en el caso de la construcción de nociones ecológicas. Investigación en la Escuela, 28, 23-36.

García, F. (1999). El medio urbano en la educación secundaria obligatoria. Las ideas de los alumnos y sus implicaciones curriculares. Tesis doctoral no publicada. Universidad de Sevilla.

García, F., y Porlán, R. (2000- Febrero). El Proyecto IRES (Investigación y Renovación Escolar) Biblio 3W. Revista Bibliográfica de Geografía y Ciencias Sociales de la Universidad de Barcelona, No. 205.

Gil, D., Carrascosa, J., y Martínez, F. (2000). Una disciplina emergente y un campo específico de investigación. En Perales, J. y Cañal, P. Didáctica de las Ciencias Experimentales. Alcoy: Marfil.

Giordan, A., y De Vecchi, G. (1995). Los orígenes del saber. De las concepciones personales a los conceptos científicos. Sevilla: Díada Editora.

Goodson, I. (1991). La construcción social del currículum, posibilidades y ámbitos de investigación de la historia del currículum. Revista de Educación. 295, 7-37.

Grupo Investigación en la escuela. (1991). Proyecto curricular "Investigación y Renovación Escolar" IRES. Vol. I. El Modelo Didáctico de Investigación en la Escuela. Vol. II. El Marco curricular. Vol. III. El currículo para la formación permanente del profesorado. Vol. IV. Investigando Nuestro Mundo. Sevilla: Díada Editora.

Habermas, J. (1986). Ciencia y técnica como "Ideología". Madrid: Tecnos.

Habermas, J. (1987). Teoría de la acción comunicativa. Racionalidad de la acción y racionalización social. Madrid. Taurus.

Izquierdo, M. (2005). Hacia una teoría de los contenidos escolares. Revista Enseñanza de las Ciencias, 23(1), 111-122.

Martínez, C. (2000). Las propuestas curriculares de los profesores sobre el conocimiento escolar: dos estudios de caso en el área del conocimiento del medio. Tesis doctoral no publicada. Universidad de Sevilla.

Martínez, C. (2005a). Las propuestas de conocimiento escolar en los inicios del aprendizaje de la química: un estudio de caso en las clases de ciencias en sexto grado de educación básica. Tolima: Centro de Investigaciones, Universidad del Tolima.

Martínez, C. (2005b). De los contenidos al conocimiento escolar. Revista Educación y Pedagogía, XVII, 43, 149-162.

Martínez, C. (2009). El conocimiento profesional de los (as) profesores (as) de ciencias: algunos aspectos centrales en el desarrollo de la línea de investigación. Revista Científica, 11, 15-23.
Martínez, C., y Martínez, V. (2004). Conocimiento escolar: de lo simple a lo complejo, una mirada desde la enseñanza de las ciencias y del arte. Trabajo presentado para el IV Encuentro Ibero-americano de colectivos Escolares y redes de profesores que hacen investigación en la escuela, Brasil.

Martínez, C., y Rivero, A. (2001). El conocimiento profesional sobre el conocimiento escolar en la clase de conocimiento del Medio. Revista Investigación en la Escuela, 45, 65-75.

Martínez, C., y Rivero, A. (2005). Algunos aspectos a considerar en una propuesta de conocimiento escolar desde una perspectiva compleja. Reflexiones en torno a un estudio de caso en las clases de ciencias. Revista Enseñanza de las Ciencias (Número extra).

Martínez, C., y Rivero, A. (2009). Las propuestas de conocimiento escolar: entre el conocimiento científico y el escolar. Revista Enseñanza de las Ciencias (Número extra).

Martínez, C., Molina, A., y Reyes, D. (2010). Conocimiento escolar en la didáctica de las ciencias: una aproximación al problema. Memorias II Congreso EDUCYT. Cali. 2010. Obtenido el 6 de Julio de 2011, desde, http://www.educyt.org/portal/images/ stories/ponencias/sala_9/9conocimiento_escolar_en_la_didactica_de_las_ciencias_una_aproximacion_al_problema.pdf

Martínez, C., y Molina, A. (2011). La especificidad del conocimiento profesional y del conocimiento escolar en las clases de ciencias: algunas relaciones con la cultura. Revista EDUCyT, 2 (2), 35-57.

Martínez, C., Valbuena, E., Molina, A., y Hedereich, C. (2009). El conocimiento profesional de los profesores de ciencias de primaria sobre el conocimiento escolar en el Distrito Capital. Proyecto de Investigación. Colciencias-Universidad Distrital Francisco José de Caldas-Universidad Pedagógica Nacional. Bogotá.

Martínez, C., Valbuena, E., Molina, A., Hedereich, C., y Reyes, D. (2011). Una hipótesis de progresión del conocimiento del profesor de ciencias sobre el conocimiento escolar. Trabajo presentado para el $\mathrm{V}$ Congreso Internacional sobre Formación de Profesores. Bogotá.

Martínez, V., y Fernández, N. (2000). El beso, una aproximación didáctica. Trabajo presentado para el Seminario doctoral: el desarrollo profesional y el conocimiento profesional en CC.SS, Universidad de Sevilla.

Martín, R. (1994). El conocimiento del cambio químico en la formación inicial del profesorado. Estudio de las concepciones disciplinares y didácticas de los estudiantes de magisterio. Tesis doctoral no publicada, Universidad de Sevilla.

Martín, R., y Rivero, A. (2001). Construyendo un conocimiento profesionalizado para enseñar ciencias en la educación secundaria: los ámbitos de investigación profesional en la formación inicial del profesorado. Revista Interuniversitaria de Formación del Profesorado, 40, 63-79.

Merchán, F. J., y García, F. (1996). El Proyecto IRES: una alternativa para la transformación escolar. Obtenido el 9 de Junio de 2011, desde, http://www.quadernsdigitals.net/datos_web/ hemeroteca/r_3/nr_39/a_619/619.htm

Mojica, L., Molina, A., López, D., y Torres, B. (2005). La formación del pensamiento científico y tecnológico de niños y niñas. Reflexiones para la construcción de una agenda regional para la región central de Colombia. Enseñanza de las ciencias (Número Extra). Obtenido el 15 de Octubre de 2009, desde, 
http://ensciencias.uab.es/webblues/www/congres2005/material/ comuni_orales/2_Proyectos_Curri/2_2/Mojica_853.pdf

Moreira, M. (2005). Una visión Toulminiana respecto a la disciplina investigación básica en Educación en Ciencias: el rol del foro institucional. Ciência \& Educação, 11 (2), 181-190.

Morín, E. (1986). El método: I. La naturaleza de la naturaleza. Madrid: Cátedra.

Morín, E. (1984). Ciencia con consciencia. Barcelona: Anthropos.

Morín, E. (1988). El Método III: El Conocimiento del Conocimiento. Madrid. Cátedra.

Morín, E. (1995). Introducción al pensamiento complejo. Barcelona: Gedisa.

Morín, E. (2000). Los siete saberes necesarios para la educación del futuro. Trabajo presentado en el Ciclo "La educación que queremos". Fundación Santillana. Obtenido el 10 de Enero de 2002, desde, www.santillana.es

Morón H., y Wamba A., (2010). La percepción sobre los riesgos ambientales como indicador de los obstáculos y dificultades para la construcción de un concepto de Medio Ambiente Responsable. Bio-grafia: escritos sobre la Biología y su Enseñanza, 3 (4). Obtenido el 25 de Marzo de 2011, desde, http://www. pedagogica.edu.co/revistas/ojs/index.php/bio-grafia/article/ viewPDFInterstitial/340/348

Nardi, M., y Almeida, M. A. (2005). Constituição da área de ensino de ciências no Brasil: memórias de pesquisadores. Enseñanza de las ciencias (Número Extra). Obtenido el 15 de Octubre de 2009, desde, http://ensciencias.uab.es/webblues/www/ congres2005/material/comuni_orales/3_Relacion_invest/3_1/ Nardi_476.pdf

Novak, J. (1988). El constructivismo humano: hacia la unidad en la elaboración de significados psicológicos y epistemológicos. En Porlán, R., García Díaz, J. E., y Cañal, P. (Comps.). Constructivismo y enseñanza de las ciencias. Sevilla: Díada.

Peme-Aranega, C., De Longhi, A., y Moreno, A. (2005). Estudio longitudinal de las creencias de una docente de física. El proceso de reflexión orientado, acerca de ellas, como mejoramiento de su práctica. Enseñanza de las ciencias (Número Extra). Obtenido el 2 de Octubre, desde, http://ensciencias.uab.es/webblues/www/ congres2005/material/comuni_orales/1_ense_ciencias/1_1/ Peme-aranega_226.pdf

Porlán, R. (1998). Pasado, presente y futuro de la didáctica de las ciencias. Enseñanza de las Ciencias, 16 (1), 175-185.

Porlán, R. (1993). Constructivismo y escuela. Hacia un modelo de enseñanza-aprendizaje basado en la investigación. Sevilla: Díada Editora.

Porlán, R. (1988). Del pensamiento a la investigación. Cuadernos de Pedagogía, 161, 22-24.

Porlán, R. (1987). El maestro como investigador en el aula. Investigar para conocer, conocer para enseñar. Investigación en la Escuela, 1, 63-70.

Porlán, R., García, D., y Cañal, P. (1988). Un enfoque constructivista e investigativo para la formación de formadores en didáctica de las ciencias. En C. Marcelo. Avances en el estudio del pensamiento de los profesores. Sevilla: Publicaciones de la Universidad de Sevilla.
Porlán, R. y Martín. (2005). ¿Qué criterio utilizamos para ordenar las Actividades? Un ejemplo de progresión de las concepciones de los estudiantes de magisterio. Trabajo presentado en el IV Encontro Ibero-Americano de colectivos escolares e redes de profesores que fazem investigacao na sua escola. Obtenido en Mayo de 2005, desde, ensino.univates.br/ 4iberoamericano/ trabalhos/trabalho301.pdf

Porlán, R., y Rivero, A. (1998). El conocimiento de los profesores. Sevilla: Díada Editora.

Quadrado, R., y Ribeiro. P. (2005). O corpo na escola: alguns olhares sobre o currículo. Enseñanza de las ciencias (Número Extra). Obtenido el 13 de Octubre de 2009, desde, http://ensciencias.uab.es/webblues/www/congres2005/material/comuni_orales/2_Proyectos_Curri/2_1/Quadrado_040.pdf

Reyes, D. (2009). El conocimiento didáctico del contenido en el profesor de física en formación inicial: el caso de la enseñanza del campo eléctrico. Tesis Doctoral no publicada. Doctorado Interinstitucional en Educación. Universidad Distrital Francisco José de Caldas.

Reyes, D., y Martínez, C. (2011). Conocimiento didáctico del contenido en profesores de física en formación inicial. Trabajo presentado en el $\vee$ Congreso Internacional sobre Formación de Profesores, Bogotá.

Rubiano, L. (2010). Estudio de las concepciones sobre la vida silvestre de estudiantes de un curso de contexto de la Universidad Nacional de Colombia. Manuscrito en preparación. Doctorado Interinstitucional en Educación. Universidad Distrital Francisco José de Caldas.

Rubiano, J., y Martínez, C. (2011). Construcción de una hipótesis de progresión para el estudio de las concepciones de estudiantes universitarios sobre la vida silvestre. Trabajo presentado en el V Congreso Internacional sobre Formación de Profesores, Bogotá.

Shulman, L. S. (2001). Conocimiento y enseñanza. Estudios públicos, 63, 163-196.

Solís, E. (2005). Concepciones curriculares del profesorado de física y química en formación inicial. Tesis Doctoral no publicada. Universidad de Sevilla.

Toulmin, S. (1977). La comprensión humana. Vol. I: el uso colectivo y la evolución de los conceptos. Madrid: Alianza Editorial.

Travé, G., Pozuelos, F., y Cañal, P. (2005). ¿Cómo enseñar investigando? Análisis de las percepciones de tres equipos docentes con diferentes grados de desarrollo profesional. Obtenido desde www.rieoei.org/deloslectores/1366Trave.pdf

Valbuena, E. (2007). El conocimiento didáctico del contenido biológico. Estudio de las concepciones disciplinares y didácticas de futuros docentes de la Universidad Pedagógica Nacional (Colombia). Madrid: Universidad Complutense. 


\section{Diálogo del conocimiento}

Resulta importante hacer una reflexión sobre los conocimientos que hacen presencia en el lugar cultural llamado Escuela, particularmente referirse a lo que los miembros de las comunidades académicas de docentes han denominado "Conocimiento Escolar", es un gran reto, por ello considero de significativo interés el artículo de las profesoras Carmen Alicia Martínez y Vilma Martínez.

El cuestionamiento con que inician su texto ¿Por qué no es suficiente el conocimiento disciplinar para ejercer el proceso de enseñanza-aprendizaje en un espacio como la escuela?, es una pregunta muy interesante que las autoras abordan y que seguramente cada lector podrá responder luego de culminar la lectura del presente artículo, y que esperamos acompañe sus reflexiones a la hora de hacer propuestas de trabajo educativo.

En el ámbito escolar nuestro magisterio esta acostumbrado a trabajar con los conocimientos disciplinares, que históricamente se han establecido como prioritarios en las instituciones educativas, estos son las ciencias naturales: física, química, biología, matemáticas, tecnologías; ciencias humanas: sociales, filosofía, literatura, español; y las bellas artes, todos vistos como conocimientos que se deben saber, como condición fundamental para poder instalarse en la era de la globalización, pero no lo estamos con los conocimientos que traen los estudiantes desde sus propias vivencias y sus contextos culturales, los cuales habitan, estos últimos son en la mayoría de las veces proscritos, se ignoran y cuando se reconocen es para trabajar en contra de ellos, como bien lo enuncia el artículo, menos aún estamos acostumbrados a trabajar con otro tipo de conocimientos a los cuales de una manera extraña hemos llamado saberes, para denotar que ellos no tienen el estatus de conocimientos y menos de conocimientos disciplinares.

En este contexto en el que nos instalan las autoras, vale aclarar que la hipótesis de progresión se debe entender, no necesariamente bajo la llana acepción de progresión, es decir, acción y efecto de progresar o de mejorar en cualquier cosa de manera consecutiva, al igual que en la progresión aritmética, sino que metodológicamente es un interesante recurso para valorar el proceso de construcción de conocimiento en las aulas, y permitir un reconocimiento del desarrollo de la propia capacidad para construir conocimientos en la escuela, por ello vale el siguiente llamado a no caer en una valoración esquemática, lineal y consecutiva de los niveles de progresión que se puedan determinar o postular desde el conocimiento docente, pues esta hipótesis de progresión metodológica puede ser asumida de manera multidireccional, en un proceso discontinuo y no necesariamente causal.

Así, uno de los valores de la hipótesis de progresión expuesta en el artículo, puede radicar en el mismo proceso que lleva al docente a postular sus matrices de nivel de formulación Vs contenidos, y la búsqueda y sistematización de las ideas expresadas por los alumnos, para catalogarlas en uno de los niveles propuestos o mejor, en la sistematización de las ideas de los estudiantes y su posterior organización en una matriz que surja de dicho proceso, ante el cual el maestro se pueda sorprender por la emergencia de niveles no sospechados que surgen de las múltiples interacciones en las clases y de los múltiples diálogos de saberes propiciados por las actividades propuestas.

Sin embargo, el texto en sus diversas referencias, de investigadores contemporáneos, sigue dejando ver el papel altamente activo en la construcción de conocimiento disciplinar y científico del conocimiento llamado común, a este se le asigna una subvaloración de simple o no riguroso. Y en el caso del pensamiento de los estudiantes en las actividades escolares; este se constituye en un punto de partida elemental, sin estructura, de menor complejidad, desde el cual los escolares deben partir para alcanzar la construcción de unos conocimientos, ellos sí, rigurosos y complejos, los cuales podemos llamar conocimiento escolar. En esta dirección, si se quiere hacer una extrapolación en el razonamiento, podrá ser valorado -los conocimiento escolares- como simples o menos complejos, frente al conocimiento del profesor y este a su vez, ser igualmente valorado como de menor rigurosidad y complejidad frente al conocimiento científico, denotado como superior; postulando una escala de menor a mayor complejidad en lo que se refiere a los conocimientos.

Discusión que reviste de importancia nuestro que hacer docente, en este sentido podemos iniciar una polémica al plantear que el conocimiento, cualquiera que sea su naturaleza común, escolar, del maestro, disciplinar científico, artístico, es solo eso; conocimiento, que no permite una estratificación o gradación, sino que todos ellos igualmente valorados dialogan de maneras particulares en las diferentes instituciones culturales y en la escuela como una de ellas, generando en sus participantes construcciones y reconstrucciones donde los diferentes actores comparten en diálogos significativos sus conocimientos. Como parece ser en las actividades artísticas llevadas a la escuela para el caso que presentan las autoras en este artículo.

Clara Inés Chaparro Susa

64 nodos y nudos / volumen 4 N. 32 / enero-junio 2012 